\title{
Fahr Disease: A Rare Disease That is Diagnosed Incidentally in the Emergency Department
}

\author{
Uzunget $\mathrm{SC}^{* 1}$, Uzunget $\mathrm{SB}^{2}$, Ertürk $\mathrm{ZK}^{3}$ and Sayın $\mathrm{R}^{4}$
}

${ }^{1}$ Health Unit, Erkunt Sanayi, Ankara, Turkey

${ }^{2}$ Department of Cardiology, Umut Hospital, Ankara, Turkey

${ }^{3}$ Department of Emergency Medicine, Etimesgut State Hospital, Ankara, Turkey

${ }^{4}$ Department of Neurology, Ufuk University Faculty of Medicine, Turkey

*Corresponding author: Uzunget SC, Health Unit, Erkunt Sanayi, Ankara, Turkey, Fax: +90 312267 0059, Tel: 05077079909, E-mail: suzunget@yahoo.com

\section{Citation: Uzunget SC, Uzunget SB, Ertürk ZK, Sayın R (2018) Fahr Disease: A Rare Disease That is}

Diagnosed Incidentally in the Emergency Department. J Case Rep Stud 6(6): 602. doi: 10.15744/2348-9820.6.602

Received Date: September 27, 2018 Accepted Date: December 29, 2018 Published Date: December 31, 2018

\begin{abstract}
Introduction: Fahr Disease (FD) is a rare disorder presenting with basal ganglia calcification and its etiology is still unknown. Although, the common cause of the disease is asymptomatic, but it might manifest with neuropsychiatric symptoms. Dizziness is one of the common symptoms at emergency services and mostly it requires advanced examination to establish a final diagnosis. When a neurological condition is suspected to cause dizziness, it is tried to be diagnosed by imaging methods such as Cranial Computed Tomography (CT) and Brain Magnetic Resonance Imaging (MRI), and knowing the relationship between calcification and symptoms will keep the patient away from unnecessary investigations.

Case Presentation: In this manuscript, we present four cases admitted by our emergency service with dizziness and diagnosed Fahr Disease by Cranial CT.

Methods: The patients who were admitted to Ufuk University Emergency Department with the complaint of dizziness between 20152017 were examined retrospectively and four patients with basal ganglia calcification were evaluated.

Conclusion: Fahr Disease should be considered in differential diagnosis at emergency services when neuropsychiatric complaints and unidentified neurological complaints have been seen in the presence of calcification that is detected by Cranial CT.

In emergency department, emergency physicians, who are obliged to establish the correct diagnosis in the shortest time and without causing harm to the patient, should be able to think of the rare diseases, besides the diseases which are common in the diagnosis process. This will keep emergency physicians away from unnecessary operations, time, labor and financial loss.
\end{abstract}

Keywords: Fahr Disease; Dizziness; Emergency Department; Calcification

\section{Introduction}

Fahr Disease (FD) is a rare disorder presenting with basal ganglia calcification and its etiology is still unknown. Clinical manifestation varies widely, although common cause of the disease is asymptomatic. It might manifest with neuropsychiatric symptoms, seizure, and dementia, extrapyramidal and cerebellar symptoms.

Basal ganglia calcification known as Fahr's Disease or Fahr Syndrome is a rare neurological disorder [1,2]. Bilateral calcification in the basal ganglia is important for diagnosis. To make a diagnosis of Fahr Syndrome; There should be bilateral calcification in cranial CT, autosomal dominant inheritance, progressive neurological dysfunction, absence of infection, toxin or drug exposure and absence of mitochondrial dysfunction [2].

Dizziness is one of the most common symptoms requiring frequent consultation of neurology at the emergency department $[3,4]$. Considering all the major symptoms, dizziness is seen in practice at thirdly and $4 \%$ of all cases have this complaint at the emergency department $[5,6]$.

Dizziness is one of the common symptoms at emergency services and mostly it requires advanced examination to establish a final diagnosis. Most of the time, it is necessary to perform further tests such as CT and MRI in order to be able to confirm these complaints in the emergency department. This situation results in some tests being performed more frequently in emergency departments. Kerber et al. showed that the use of CT / MRI increased by 160\% from 1995 to 2004 [4]. Emergency physicians, who act quickly with the motivation of deciding the problem immediately, sometimes encounter with unexpected results in their 
examinations and have to solve a new problem. Establishing the association of symptoms or patient complaints with unexpected results may reduce the workforce of emergency physicians.

In this manuscript, we present four cases admitted by our emergency service with dizziness and diagnosed Fahr Disease by Cranial CT.

\section{Case Reports}

\section{Case 1}

A thirty-eight years old woman referred our emergency service with pre-syncope. She complained about dizziness with transient unconsciousness occurring a few times a day in the last week period. She did not have familial and past medical history. Her physical examination was unremarkable. Laboratory investigations revealed hemoglobin ( $\mathrm{Hb}$ ) hematocrit (Hct), MCV, Platelet, Urea, Creatinine, ALT, AST and GGT tests were in normally range. Calcium was $7.8 \mathrm{mg} / \mathrm{dl}$ (reference range: 8,3-10.5 mg/dl). Cranial CT of the patient showed symmetrical calcification affecting the bilateral basal ganglia.

\section{Case 2}

A sixty years old woman admitted to the emergency service with dizziness. The patient has this complaint constantly and it was not involved in movement. Her resume had an operation story for adrenal adenoma. Vital findings were normal, neurological and physical examinations were normal. Hb, Hct, MCV, Platelet, Urea, Creatinine, AST, ALT, GGT and Calcium were in normal range. Symmetric calcification that affecting the basal ganglia were seen in the Cranial CT.

\section{Case 3}

A forty-eight years old male patient was admitted to our emergency service with dizziness and slight nausea for 2 hours. There was no other complaint. He did not have familial and past medical history. His physical examination was unremarkable, vital findings were stable. Hb, Htc, MCV, Platelet, Urea, Creatinine, AST, ALT, GGT and Ca tests were in normal range. Symmetric calcification affecting the basal ganglia were seen in the Cranial CT of the patient.

\section{Case 4}

A Sixty-three years old woman admitted to our emergency service with a daytime headache and dizziness. The patient had a moderate, widespread headache. She has no other neurological complaints such as nausea, vomiting, photophobia. She has hypertension and gout and renal transplantation in 2005 and coronary by-pass in 2016. His physical examination was unremarkable. $\mathrm{Hb}, \mathrm{Htc}, \mathrm{MCV}$, Platelet, Urea, Creatinine, AST, ALT, GGT and Ca were in normal range. Cranial CT of the patient showed bilateral symmetric calcification affecting the basal ganglia and thalamus.

\section{Methods}

Between 2015-2017, Ufuk University emergency room was examined retrospectively. Patients with basal ganglia calcification was evaluated with Hb, Htc, MCV, Platelet, Urea, Creatinine, AST, ALT, GGT and Ca test results.

\section{Discussion}

The Fahr's disease may be asymptomatic or with neurological and psychiatric disorders. Close to half of cases arise with neurological findings and basal ganglia calcification. The most common neurological findings are headache, dizziness, movement impairment, syncope, and seizures. In addition, dementia, parkinsonism, paresis, tremor, chorea, walking and speech disorders are also seen. We had one pre-syncope and headache with dizziness. All of the cases had normal neurological examination and the only pathologic and important finding was bilateral calcification seen on CT.

Treatment of Fahr Disease is about reducing symptoms and improving quality of life [7]. It should be noted, however, that there is no disorder associated with calcium metabolism in the patient [8]. We presented with a complaint of dizziness in 4 cases that there was no other reason to explain the pathology in the examinations, but we found bilateral basal ganglia calcification in the CT.

The fact that rapid decision-making and the need to reach the result quickly in Emergency Departments lead physicians to be defensive. The evaluation of different diseases at the same time and different group of diseases considered in the differential diagnosis leads to an increase in examination requests. Saber Tehrani et al. showed that the financial cost for ED patients with Acute Vestibular Syndrome is rising over time, in part driven by neuroimaging [9]. In fact, simple algorithms can be used to determine if dizziness is central or peripheral in the evaluation of emergency cases because some neurological disorders causing dizziness require rapid treatment and interdisciplinary approach [10,11]. We know that dizzy patients presenting to an emergency department (ED) would have been dangerous, treatable diseases [12]. For this reason, physical examination, laboratory tests and brain imaging should be combined. Some unexpected findings may remove the emergency physician from the patient's main complaint during examinations. For this reason, knowing the relationship between calcification and symptoms will keep us and the patient away from unnecessary investigations. 


\section{Conclusion}

As a result, Fahr Disease should be considered in differential diagnosis at emergency services when neuropsychiatric complaints and unidentified neurological complaints have been seen in the presence of calcification that are detected by Cranial CT.

\section{References}

1. Mufaddel AA, Al-Hassani GA (2014) Familial idiopathic basal ganglia calcification (Fahr's disease). Neurosciences 19: 171-7.

2. Saleem S, Aslam HM, Anwar M, Anwar S, Saleem M, et al. (2013) Fahr's syndrome: literature review of current evidence. Orphanet J Rare Dis 8: 156.

3. Navi BB, Kamel H, Shah MP, Grossman AW, Wong C, et al. (2012) Rate and predictors of serious neurologic causes of dizziness in the emergency department. Mayo Clin Proc 87: 1080-8.

4. Kerber KA, Meurer WJ, West BT, Fendrick AM (2008) Dizziness presentations in US Emergency Departments, 1995-2004. ACAD EMERG MED 15: 744-50.

5. Spiegel R, Kirsch M, Rosin C, Rust H, Baumann T, et al. (2017) Dizziness in the emergency department: an update on diagnosis. Swiss Med Wkly 147: w14565.

6. Ahsan SF, Syamal MN, Yaremchuk K, Peterson E, Seidman M (2013) The costs and utility of imaging in evaluating dizzy patients in the emergency room. Laryngoscope 123: 2250-3.

7. Otu AA, Anikwe JC, Cocker D (2015) Fahr's disease: a rare neurological presentation in a tropic setting, Clin Case Rep 3: 806-8.

8. Shahidi GA, Safdarian M (2017) Fahr Disease: Idiopathic basal ganglia calcification. Iran J Neurol 16: 53-4.

9. Saber Tehrani AS, Coughlan D, Hsieh YH, Mantokoudis G, Korley FK, et al. (2013) Rising annual costs of dizziness presentations to U.S. emergency departments. Acad Emerg Med 20: 689-96.

10. Walther LE (2017) Current diagnostic procedures for diagnosing vertigo and dizziness. GMS Curr Top Otorhinolaryngol Head Neck Surg 16: 10.3205/ cto000141.

11. Edlow JA (2013) Diagnosing Dizziness: We are teaching the wrong paradigm. Acad Emerg Med 20: 1064-6.

12. Newman-Toker DE, Hsieh YH, Camargo CA, Pelletier AJ, Butchy GT, et al. (2008) Spectrum of dizziness visits to US emergency departments: crosssectional analysis from a nationally representative sample. Mayo Clin Proc 83: 765-75.

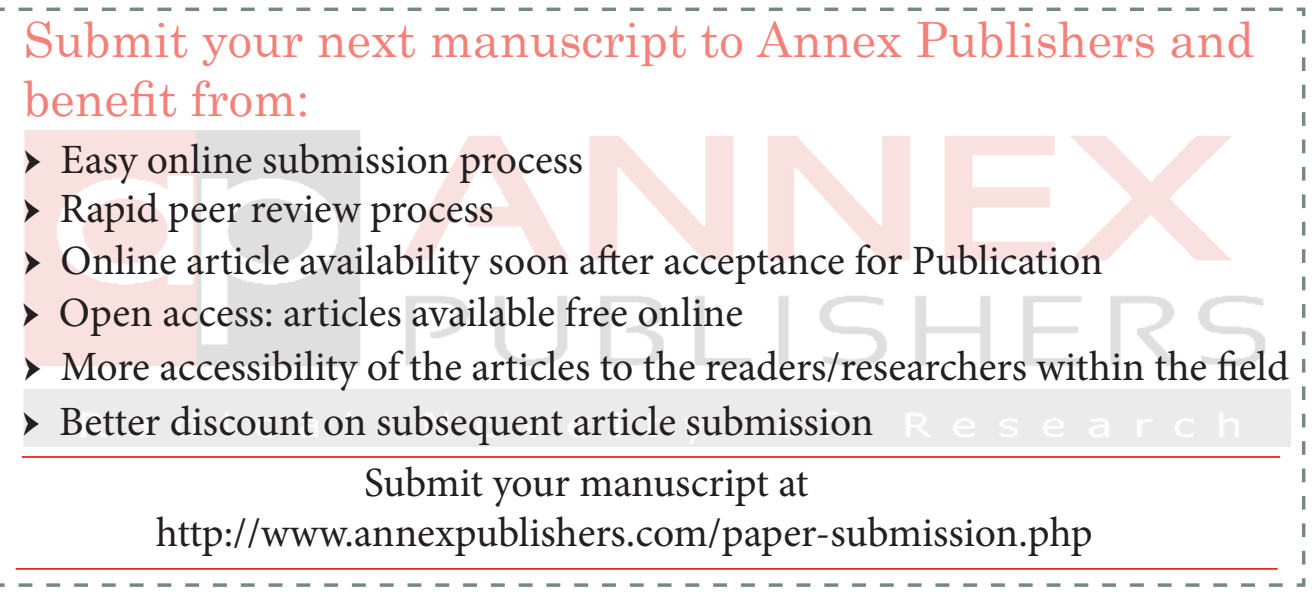

